\title{
All non-dopant red-green-blue composing white organic light-emitting diodes
}

\author{
Shi-Jay Yeh ${ }^{\text {a }}$, Hung-Yang Chen ${ }^{\text {a }}$, Min-Fei Wu ${ }^{\text {a }}$, Li-Hsin Chan ${ }^{\text {a }}$, \\ Chih-Long Chiang ${ }^{a}$, Hsiu-Chih Yeh ${ }^{a}$, Chin-Ti Chen ${ }^{\text {a,* }}$, Jiun-Haw Lee ${ }^{\text {b,* }}$ \\ a Institute of Chemistry, Academia Sinica, Taipei 11529, Taiwan, ROC \\ ${ }^{\mathrm{b}}$ Graduate Institute of Electro-Optical Engineering and Department of Electrical Engineering, National Taiwan University, \\ Taipei 10617, Taiwan, ROC
}

Received 2 August 2005; received in revised form 10 December 2005; accepted 13 December 2005

Available online 11 January 2006

\begin{abstract}
All non-dopant white organic light-emitting diodes (WOLEDs) have been realized by using solid state highly fluorescent red bis(4-( $N$-(1-naphthyl)phenylamino)phenyl)fumaronitrile (NPAFN) and amorphous bipolar blue light-emitting 2-(4-diphenylamino)phenyl-5-(4-triphenylsilyl)phenyl-1,3,4-oxadiazole (TPAOXD), together with well known green fluorophore tris(8-hydroxyquinolinato)aluminum $\left(\mathbf{A l q}_{\mathbf{3}}\right)$. The fabrication of multilayer WOLEDs did not involve the hardto-control doping process. Two WOLEDs, Device B and C, different in layer thickness of $\mathbf{A l q}_{\mathbf{3}}, 30$ and $15 \mathrm{~nm}$, respectively, emitted strong electroluminescence (EL) as intense as $25,000 \mathrm{~cd} / \mathrm{m}^{2}$. For practical solid state lighting application, EL intensity exceeding $1000 \mathrm{~cd} / \mathrm{m}^{2}$ was achieved at current density of $18-19 \mathrm{~mA} / \mathrm{cm}^{2}$ or driving voltage of $6.5-8 \mathrm{~V}$ and the devices exhibited external quantum efficiency $\left(\eta_{\text {ext }}\right)$ of $2.6-2.9 \%$ corresponding to power efficiency $\left(\eta_{\mathrm{P}}\right)$ of $2.1-2.3 \mathrm{~lm} / \mathrm{W}$ at the required brightness. The thickness of $\mathbf{A l q}_{\mathbf{3}}$ layer is decisive in color quality of non-dopant WOLEDs. The Commission Internationale de l'Eclairage (CIE) coordinates of fairly white EL of Device B varied only little from $(0.34,0.39)$ to $(0.34,0.38)$ at driving voltage between 6 and $14 \mathrm{~V}$. Device B exhibited relatively high color rendering indexes (CRIs) in the range of 74-81, which were essentially voltage-independent. The other WOLED, Device C, showed even better color purity of white $\mathrm{EL}\left(\mathrm{CIE}_{x, y}=0.34,0.31\right)$ along with higher $\mathrm{CRI}$ of 83 at $8 \mathrm{~V}$, although higher voltage deteriorated the color quality of WOLED.
\end{abstract}

(C) 2005 Elsevier B.V. All rights reserved.

PACS: 71.20.Rv; 72.80.Le; 73.61.Ph

Keywords: White; Organic light-emitting diode; Non-dopant; Red; Blue

\footnotetext{
* Corresponding authors. Tel.: +886 227898542; fax: +886 227831237.

E-mail addresses: cchen@chem.sinica.edu.tw (C.-T. Chen), jhlee@cc.ee.ntu.edu.tw (J.-H. Lee).
}

\section{Introduction}

Organic light-emitting diodes (OLEDs) have rapidly developed over the last decade and have become the highly competitive alternative of full color flat panel display [1-5], which is currently 
dominated by the liquid-crystal display (LCD) [6]. Besides glamorous displays, an intriguing and potentially energy-saving application for OLEDs are decorative or domestic solid-state lighting (SSL) and the flat panel display backlight [6-10], OLEDs for SSL or back light for flat panel display have not been considered to be realistic until recent discovery of highly efficient organometallic phosphorophores $[11,12]$. The introduction of electrophosphorescence moves the upper limit of the power efficiency $\left(\eta_{\mathrm{P}}\right)$ from $\sim 20 \mathrm{~lm} / \mathrm{W}$ for electrofluorescent materials to $\sim 80 \mathrm{~lm} / \mathrm{W}$, which surpasses $15 \mathrm{~lm} / \mathrm{W}$ for incandescent light bulb and is comparable with that of a fluorescent lamp. With exceptions of low efficient singlet exciplexes or triplet excimers emitting broad-band (white) emission, satisfactory white organic light-emitting diodes (WOLEDs) are always constructed on a multilayer device structure with two (green blue/orange red or blue/yellow) to three (red, green, and blue) light-emitting components that can be fluorophores and/or phosphorophores [12-31]. Among these color components of WOLEDs, many blue, most of yellow, and nearly all orange to red fluorophores suffer from a common problem, namely the concentration quenching of fluorescence in solid state. Consequently, the dopant light-emitter of guest-host system becomes a universal method for solving the quenching problem of these long-wavelength, orange to red fluorophores [32]. Emission quenching due to the long lifetime of the triplet state (phosphorescence) causes severe triplet-triplet annihilation of electroluminescence (EL) which is significant even at medium current density greater than $50 \mathrm{~mA} / \mathrm{cm}^{2}$ for most cases. Doping is also an inevitable solution to all colors of phosphorophores. Therefore, the doping process seems to be a must in the fabrication of WOLEDs based on small molecular materials, either fluorophores or phosphorophores. However, in practical OLED manufacture, the doping process is not a trivial task to handle, considering the reproducibility of the optimum doping level, which is normally low and less than 1-2\% and let along that it should be carefully controlled in a narrow effective range of $\pm 0.5 \%$ for a consistent performance of the devices.

Recently, we have successfully developed a few bright (electroluminance $(L)$ of $8000-12,400 \mathrm{~cd} / \mathrm{m}^{2}$ ) and efficient (external quantum efficiency, $\eta_{\text {ext }}$, of $2.4-3.6 \%$ or $\eta_{\mathrm{P}}$ of $\left.0.9-1.8 \mathrm{~lm} / \mathrm{W}\right)$ non-doped red OLEDs based on extraordinary red light-emitting materials [33-36]. NPAFN (Scheme 1) is one of those rare materials that have an unusual property

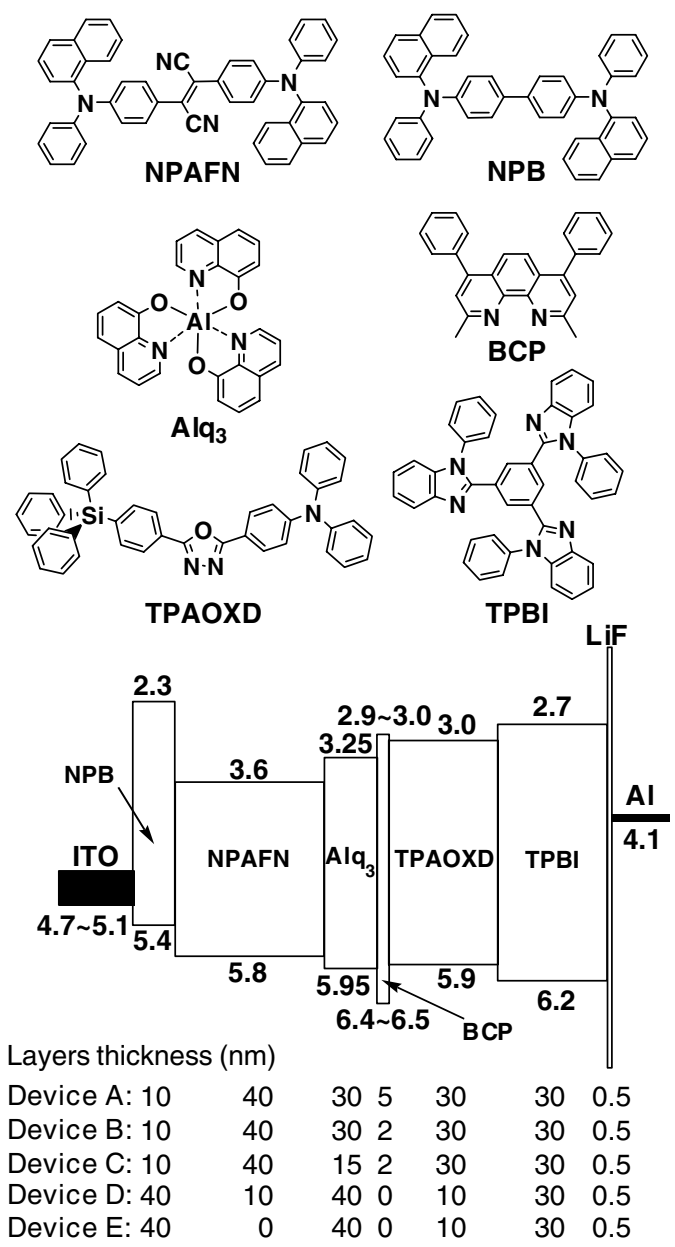

Scheme 1. Chemical structures and their relative energy level (HOMO and LUMO) diagram of NPAFN, Alq $\mathbf{q}_{3}$, TPAOXD, NPB, BCP, and TPBI. Layer structures of Device A-E are also schematically shown here with the layer thickness indicated therein.

of aggregation-induced emission (AIE) and it was adopt here as non-dopant red light-emitting material for WOLEDs [37]. In addition to NPAFN, we have also explored a new type of blue fluorophore, TPAOXD (Scheme 1), which is bipolar in nature but is an authentic amorphous molecular material [38]. High-performance (maximum brightness $\sim 20,000 \mathrm{~cd} / \mathrm{m}^{2}$ and maximum external quantum efficiency $\sim 2.4 \%$ ) blue (1931 Commission Internationale de L'Eclairage, $\mathrm{CIE}_{x, y}$ of $x=0.16$ and $y=0.18$ ) OLEDs containing TPAOXD as the non-dopant blue emitter was found to be relatively stable with little decay of external quantum efficiency at $\sim 2.3 \%$ from low to high current density $\left(10-500 \mathrm{~mA} / \mathrm{cm}^{2}\right)$ [39]. In this work, three devices $\mathrm{A}, \mathrm{B}$, and $\mathrm{C}$, were fabricated to test the idea of 
bright and efficient all non-dopant WOLEDs using NPAFN, $\mathbf{A l q}_{3}$, and TPAOXD for red, green, and blue emitters, respectively (see photoluminescence, PL, spectra in Fig. 1).

There was one preceding report about "nondoped-type" WOLED by Tsuji et al. [40].
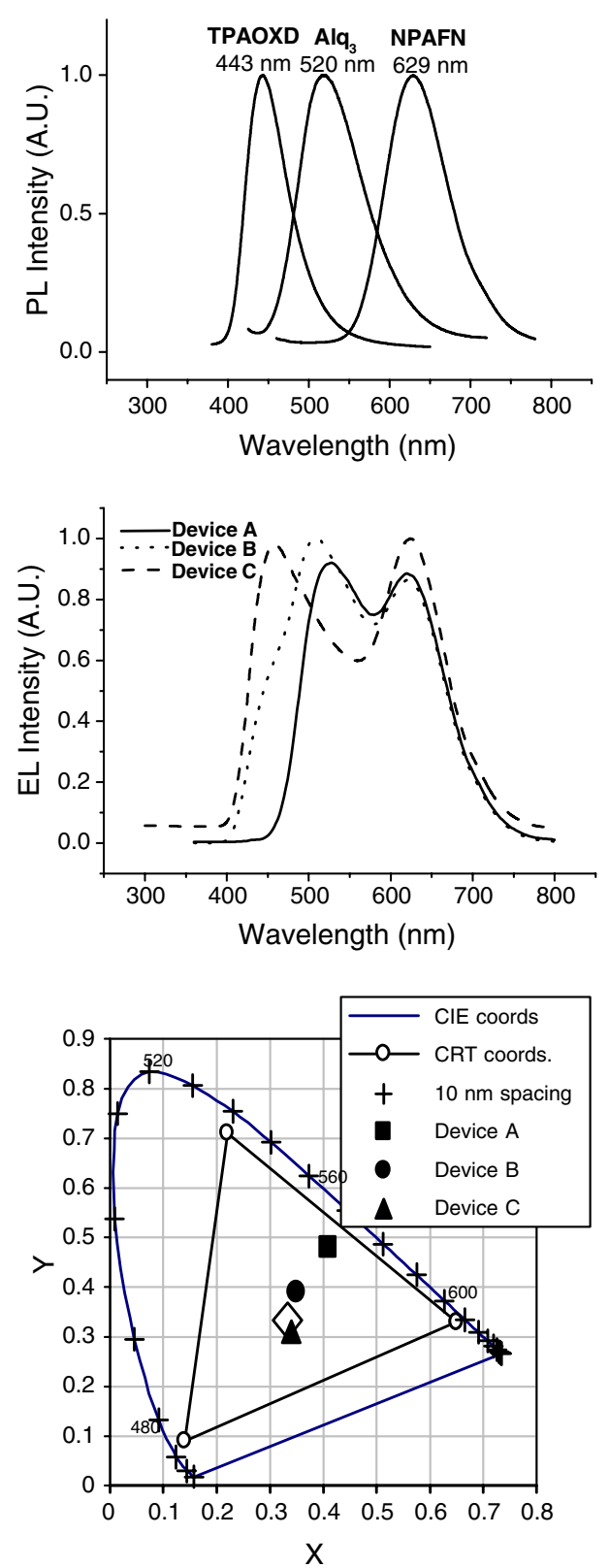

Fig. 1. Normalized photoluminescence spectra of vacuum thermal deposited thin films of TPAOXD, Alq $\mathbf{q}_{3}$, and NPAFN; EL spectra (center) and 1931 CIE coordinates (bottom) of Device $\mathrm{A}-\mathrm{C}$ at driving voltage of $8 \mathrm{~V}$. CRT (cathode ray tube) coordinates mean the standard red-green-blue color coordinates of CRT screen (traditional color television screen).
However, they were utilizing complementary twocolor system, blue emission (445 and $480 \mathrm{~nm}$ ) from NPB and orange emission $(558 \mathrm{~nm})$ from DCM (4-(dicyanomethylene)-2-methyl-6-( $p$-dimethylaminostyryl)-4H-pyran), in composing white emission. Furthermore, their WOLEDs adopt an ultrathin layer (only $1 \mathrm{~nm}$ thickness) of DCM as one of the complementary colors. Such a thin layer is unlikely to make DCM to be a "nondoped type" emitter (more likely to be a blending of DCM and NPB) and hence unlikely the "nondoped type" WOLED. This is because the authentic "nondoped type" DCM (neat DCM) emitter should have a deep red emission at wavelength greater than $620 \mathrm{~nm}$ instead of orange $558 \mathrm{~nm}$ [32,41]. Furthermore, their WOLED is weakly electroluminescent (maximum luminance $\sim 1000 \mathrm{~cd} / \mathrm{m}^{2}$ ) due to the dim blue NPB and concentration quenching orange DCM. The EL efficiency of such WOLED is expected to be low, which in fact is not available from the report.

\section{Experimental}

Device A-E were fabricated by sequential thermal vacuum deposition of thin layer of organic materials and $\mathrm{LiF}-\mathrm{Al}$ as the final cathode electrode on ITO (indium-thin-oxide)-coated glass substrate. The configuration of these devices is depicted in Scheme 1 . The current, voltage, and light intensity measurements have been described before [39]. All measurements, including the recording of EL spectra, were carried out at room temperature under ambient condition without the encapsulation of the devices. Whereas literature known figures were adopt for $\mathbf{A l q}_{\mathbf{3}}, \mathbf{B C P}$, and TPBI, HOMO energy levels of NPB, NPAFN, and TPAOXD were measured with a low-energy photo-electron spectrometer (Riken-Keiki AC-2). The addition of the absorption on-set energy to the HOMO energy levels led to LUMO energy levels of these materials. The absorption on-set energy was estimated from the solution UV-visible absorption spectra. In CRI calculation, eight test-color samples were chosen followed the definition of DIN 6169 standards. CIE 1976 $\left(L^{*} u^{*} v^{*}\right)$ color-difference formula was used to determine the resultant color shift [42]. In order to demonstrate the easy control of the white color EL from these all non-dopant devices, we fixed the thickness of NPB, NPAFN, TPAOXD, and TPBI layers at 10, 40, 30 and $30 \mathrm{~nm}$, respectively, in Device A, B, and $\mathrm{C}$, although these can be the adjustable parameters in optimizing performance of the devices. By just 
varying the thickness of $\mathbf{A l q}_{\mathbf{3}}$ and $\mathbf{B C P}$ layers we were able to tune the color of the devices showing CIE coordinates as close as to $(0.33,0.33)$, a standard figure for color-balanced WOLED.

\section{Results and discussion}

Device A is our testing model and is not a colorbalanced WOLED since the $5 \mathrm{~nm}$ layer of holeblocking BCP (2,6-dimethyl-4,7-diphenyl-1,10-phenanthroline) is simply too much for WOLED. In current case, $5 \mathrm{~nm}$ layer of BCP effectively blocks the hole from entering TPAOXD and hence limits the charge recombination and/or exciton diffusion on it. This greatly prevents the blue emission $\left(\lambda_{\max } \sim 460 \mathrm{~nm}\right)$ from TPAOXD in EL spectra (Device A in Fig. 1). Reducing the thickness of BCP layer to half as in Device B turned out to be just fine for white EL of Device B. CIE coordinates of it is $(0.35,0.39)$, which is a respectful improvement of white EL from $(0.41,0.48)$ of CIE coordinates of yellow-orange Device A (Fig. 1). Even higher color purity of white EL has been achieved by adjusting the thickness of $\mathbf{A l q}_{\mathbf{3}}$ layer. In Device $\mathbf{C}$, the thickness of $\mathbf{A l q}_{\mathbf{3}}$ layer was further reduced to half $(15 \mathrm{~nm})$ from $30 \mathrm{~nm}$ of Device B. Having both thin layers of green light-emitting $\mathbf{A l q}_{\mathbf{3}}$ and hole-blocking BCP, Device $\mathrm{C}$ showed significant increase of the blue emission from TPAOXD as well as the decrease of green emission from $\mathbf{A l q}_{\mathbf{3}}$ (Fig. 1). Such changes of relative contribution of emission color rendered virtually white EL of Device $\mathrm{C}$ as indicated by the CIE coordinates $(0.34,0.31)$ (Fig. 1). In principle, all non-dopant WOLED with any CIE coordinates as close as to $(0.33,0.33)$ can be achieved with the variation of the thickness of $\mathbf{A l q}_{\mathbf{3}}$ and $\mathbf{B C P}$ layers.

WOLEDs reached $1000 \mathrm{~cd} / \mathrm{m}^{2}$ of EL intensity, the general requirement for SSL application, at a reasonable current density range of $18-19 \mathrm{~mA} / \mathrm{cm}^{2}$ (Fig. 2), which corresponded to 6.5-8 V for Devices $\mathrm{B}$ and $\mathrm{C}$. The maximum EL intensity of 25,000 $\mathrm{cd} / \mathrm{m}^{2}$ was observed for Device B at $790 \mathrm{~mA} / \mathrm{cm}^{2}$ and $15 \mathrm{~V}$. At low current density near $0.1 \mathrm{~mA} /$ $\mathrm{cm}^{2}$, high $\eta_{\mathrm{P}}$ over $5 \mathrm{~lm} / \mathrm{W}$ were observed for Device $\mathrm{C}$ and slightly lower $4.0-4.5 \mathrm{~lm} / \mathrm{W}$ were found for Device B, although both devices display a roll-off of $\eta_{\mathrm{P}}$ at elevated current density (Fig. 2). However, high $\eta_{\text {ext }} \mathrm{s}$ of $2.9-3.3 \%$ remained relatively stable with only slight decay to $2.6-2.9 \%$ (corresponding to $2.1-2.3 \mathrm{~lm} / \mathrm{W}$ ) when the current density up to $20 \mathrm{~mA} / \mathrm{cm}^{2}$ (or $1000 \mathrm{~cd} / \mathrm{m}^{2}$ lighting intensity). Even
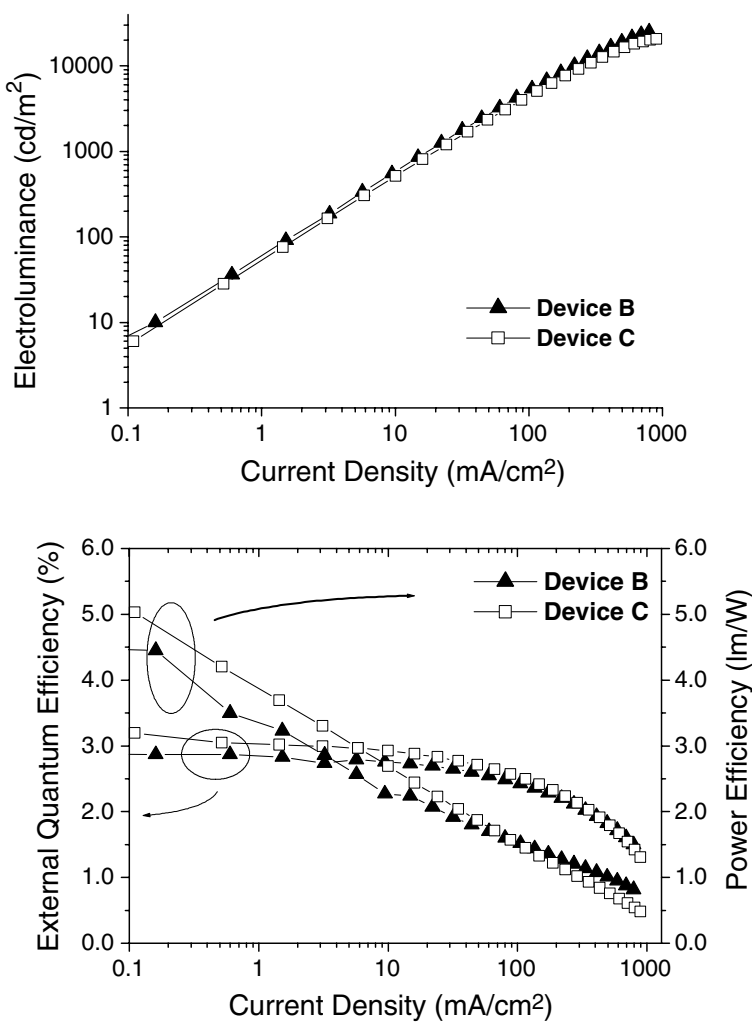

Fig. 2. Electroluminance $(L)$-current density $(I)$-efficiency $\left(\eta_{\text {ext }}\right.$ and $\left.\eta_{\mathrm{P}}\right)$ characteristics of Device B and C.

at relatively high current density of $100 \mathrm{~mA} / \mathrm{cm}^{2}$ (where WOLED showed EL intensity of $\sim 5000 \mathrm{~cd} / \mathrm{m}^{2}$, Fig. 2), $\eta_{\text {ext }} \mathrm{s}$ stayed reasonably high around $2.4-2.6 \%$. The rather current-stable external quantum efficiency can be partially attributed to the relatively stable blue emitter TPAOXD. Similar stability of $\eta_{\text {ext }}$ was previously known for blue OLEDs based on amorphous TPAOXD [39]. Containing both electron-poor oxadiazole and electron-rich triphenylamine moieties, bipolar TPAOXD possesses electro-transporting characteristic, which is crucial for a good performance WOLEDs here. We have fabricated the same WOLED by using XTPS (trans-4-(bis(2,4-dimethylphenyl)amino)-2', $3^{\prime}, 4^{\prime}, 5^{\prime}$ tetraphenylstilbene), another highly efficient (maximum external quantum efficiency up to $4.1 \%$ ) non-dopant blue light $\left(\lambda_{\max }^{\mathrm{EL}} \sim 462 \mathrm{~nm}\right)$ emitter [43]. However, the XTPS-containing WOLEDs performed poorly. Unlike bipolar TPAOXD, XTPS is stilbene-type triarylamine and preferentially transports hole instead of electron. This is not appropriate for using XTPS as blue light-emitting material that is insufficient for electron-transporting in current devices. 
Device B was unusually stable regarding CIE coordinates and CRIs. The CIE coordinates of Device B were virtually unchanged with CIE coordinates $x=0.34-0.35$ and $y=0.38-0.39$ at the driving voltage range of 6-14 V (Fig. 3). CRIs of Device B were rather high in the range of 74-81 and they also remained reasonably steady in the driving voltage between 6 and $14 \mathrm{~V}$ (Fig. 3). However, we noted that Device $\mathrm{C}$ was less stable considering either CIE coordinates or CRIs. With increasing driving voltage, CIE coordinates of Device $\mathrm{C}$ shifted to smaller figures and CRIs dropped to smaller numbers from 83 to less than 50 (Fig. 3). This is in sharp contrast to Device B. Furthermore, Device C exhibited complicate up and down of CIE coordinates and CRIs when driving voltage varied from 6 to $14 \mathrm{~V}$. Usually, such nonlinear changes imply that there are at least two different processes that are responsible for the color changes of devices. For Device C, blue EL first increased in intensity relative to the red EL (CIE coordinates changed to smaller figures) when the driving voltage was raised from 6 to $12 \mathrm{~V}$ (Fig. 3). Significant decrease of blue EL was then observed when driving voltage was further up to $14 \mathrm{~V}$ (Device $\mathrm{C}$ in Fig. 3). It is not so clear at the moment that why Device B is more stable in general than Device C. We can surmise that it is probably due to the instability of blue TPAOXD because the device having thinner $\mathbf{A} \mathbf{~ q q}_{\mathbf{3}}$ tends to have more portion of blue EL from TPAOXD (see below).

Among several possible causes for color changes, we can assume that the shifting of the charge recombination zone and/or exciton diffusion toward cathode side of the device under higher driving voltage as one of the possibilities. Such zone shifting and/ or exciton diffusing takes place in both Device B and $\mathrm{C}$ but the thick $\mathbf{A l q} \mathbf{q}_{\mathbf{3}}$ layer $(30 \mathrm{~nm})$ in between NPAFN and TPAODX layers taking up large portion of it that alleviate most of the color changes in Device B. The second possible process that changes the color of the device is the potential degradation of blue emitter TPAODX, particularly, under high voltage of $14 \mathrm{~V}$ (corresponding to very high current density $\sim 1000 \mathrm{~mA} / \mathrm{cm}^{2}$ ), which has been identified before [39]. The lowering CRIs of Device $\mathrm{C}$ with rising voltage is mainly due to the diminished relative contribution of green EL from $\mathbf{A l q}_{\mathbf{3}}$ as the charge recombination zone shifting or exciton diffusing toward blue emitter TPAODX. At high voltage of $14 \mathrm{~V}$, the relative intensity of the blue emission from TPAODX became weaken-
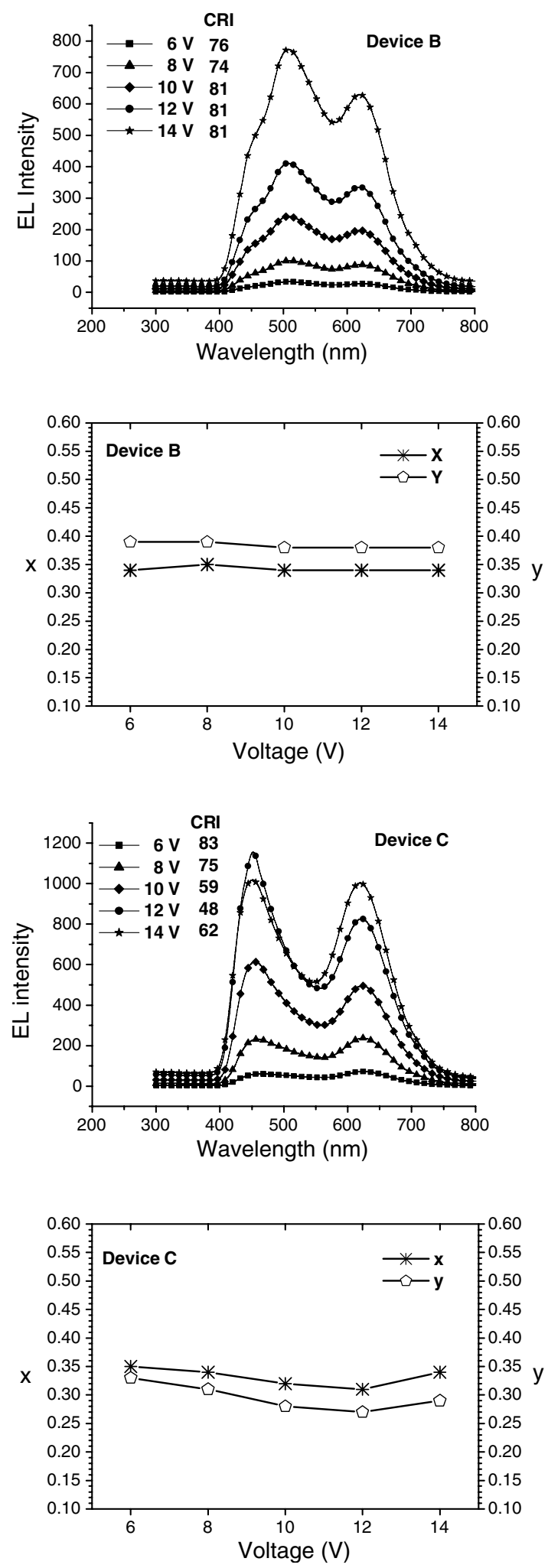

Fig. 3. The voltage dependency of EL spectra and CIE coordinates $(x, y)$, and CRIs of Device $\mathrm{B}$ and $\mathrm{C}$ under different voltage of $6,8,10,12$, and $14 \mathrm{~V}$. 
ing and the profile of white EL spectrum became less dented, which turned the CRI to a larger figure.

Among several vital issues mentioned above, the success of such WOLED depends on the appropriate adjustment of the relative EL intensity of redgreen-blue three components via the thickness control of individual light-emitting layer. Through the organ-red dye-doped spacial sensing layer in $\mathbf{A l q} \mathbf{q}_{\mathbf{3}}$, it has been demonstrated before by Tang et al. that charge-recombination is confined about $5 \mathrm{~nm}$ near the hole-transport interface in the bilayer OLED [40]. At a glance, observing the blue EL from TPAOXD from our devices, particularly Device B and $\mathrm{C}$, was rather unbelievable because of the thickness of $\mathbf{A l q}_{\mathbf{3}}$ layer is way beyond $5 \mathrm{~nm}$ and it is 30 and $15 \mathrm{~nm}$, respectively. However, we have recognized that Tang et al. also had reported that EL emission zone in the undoped $\mathbf{A l q} \mathbf{q}_{\mathbf{3}}$ system was considerably larger than the charge-recombination zone due to the exciton diffusion. The migration of the exciton was carefully estimated by Tang et al. to be more than $20 \mathrm{~nm}$ in undoped $\mathbf{A l q}_{3}$ [42]. In order to get a clear picture of the thickness-dependent EL of all non-dopant devices here, we have fabricated two more devices, Device D and E. These devices are similar to Device $\mathrm{A}-\mathrm{C}$ but $\mathbf{A l q}_{\mathbf{3}}$ layer is thicker $(40 \mathrm{~nm})$ and we remove $\mathbf{B C P}$ hole-blocking layer to simplify the issue (Scheme 1). Particularly, Device E contains no red NPAFN and we see only strong EL from $\mathbf{A l q}_{\mathbf{3}}$ accompanying with weak emission side band around $440 \mathrm{~nm}$, which strongly resembles the blue EL from TPAOXD (Fig. 4). The result of Device $\mathrm{E}$ indicates that the charge-recombination zone and/or exciton diffusion is beyond the whole $40 \mathrm{~nm}$ thick $\mathbf{A l q} \mathbf{q}_{3}$ layer and entering blue TPAOXD layer. The result is somewhat reconfirmed what Tang et al. found earlier, although $40 \mathrm{~nm}$ was a

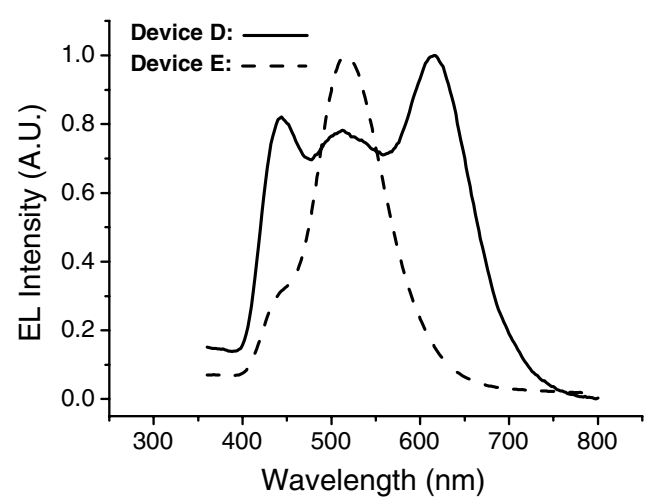

Fig. 4. EL spectra of Device D and E under voltage of $8 \mathrm{~V}$. bit beyond the estimated exciton diffusion length $\left(\geqslant 20 \mathrm{~nm}\right.$ ) for $\mathbf{A l q}_{\mathbf{3}}$. Here, according to the current results, we have a new proposal that the zone of charge-recombination and/or the range of exciton diffusion of non-doped $\mathbf{A l q} \mathbf{q}_{\mathbf{3}}$ depend on the HOMO energy level of hole-transport layer adjacent to the $\mathbf{A l q}_{\mathbf{3}}$. This is based on the result of Device D, which has a similar structure to Device E except a thin layer $(10 \mathrm{~nm})$ of red NPAFN inserted in between NPB and $\mathbf{A l q} \mathbf{q}_{\mathbf{3}}$. In addition to the strong EL from red NPAFN around $615 \mathrm{~nm}$, when compared with Device D, a significant increase of blue EL from TPAOXD relative to the green EL from $\mathbf{A l q}_{\mathbf{3}}$ was observed (Fig. 4). NPAFN has HOMO energy level of $5.8 \mathrm{eV}$, which is $0.4 \mathrm{eV}$ lower than NPB HOMO energy level (Scheme 1). NPAFN has been proven to be an efficient non-dopant red-light emitter and hole-transport material as well in the non-dopant red OLEDs [34]. We assume that the closer of the HOMO energy level of hole-transport material to that of $\mathbf{A l q}_{\mathbf{3}}$ is, the deeper of the charge recombination zone is inside $\mathbf{A l q}_{\mathbf{3}}$. Assuming the same range of exciton diffusion (a function of diffusion coefficient and the lifetime) [40], the closer charge recombination zone to the interface of $\mathbf{A l q}_{\mathbf{3}}$ and TPAOXD layers will presumably facilitates the exciton taking place in the blue light-emitting TPAOXD layer.

\section{Conclusion}

In summary, we have studied the EL properties of a series of multilayer OLEDs containing all non-dopant emitters. Devices with the structure configuration of ITO/NPB/NPAFN/Alq $/ \mathbf{A C P} /$ TPAOXD/TPBI/LiF/Al emit color-balanced white EL. The success of the all non-dopant WOLEDs here hinges on the unusual AIE red emitter of NPAFN as well as the amorphous bipolar blue emitter TPAOXD. Green light-emitting $\mathbf{A l q} \mathbf{q}_{3}$ also plays a crucial role of white color purity and stability of non-dopant WOLEDs. Under the condition of sufficient amount of blue EL contributed to the color-balanced WOLED, such as Device B and C, we propose that a thick $\mathbf{A l q}_{\mathbf{3}}$-containing device can cover most of the charge-recombination zone and reduce the number of exciton migrating into blue TPAOXD. The relatively unstable blue EL from TPAOXD is limited and CIE- or CRI-stability can thus be enhanced. WOLEDs, particularly the thick (30 nm) Alq $\mathbf{3}$-containing Device B, has been demonstrated to show satisfactory performances, including EL intensity, efficiency, CRIs, and voltage-stable 
white EL, which are comparable with or better than conventional electrofluorescence-based WOLEDs that are exclusively based on hard-to-control doping process.

\section{Acknowledgements}

This work was supported by Academia Sinica and National Science Council. We thank Prof. $\mathrm{Yu}$-Tai Tao for his instruction in the fabrication and measurement of OLEDs.

\section{References}

[1] S.M. Kelly, Flat Panel Display: Advanced Organic Materials, Royal Society of Chemistry, Cambridge, 2000.

[2] B. Johnstone, Technol. Rev. 104 (September) (2001) 80.

[3] O. Gelson, Opt. Laser Eur. 107 (June) (2003) 33.

[4] W.E. Howard, Sci. Am. 290 (February) (2004) 76.

[5] J.R. Sheats, J. Mater. Res. 19 (2004) 1974.

[6] B. Yang, Inf. Disp. 21 (February) (2005) 22.

[7] A. Bergh, D. Craford, A. Duggal, R. Haitz, Phys. Today 54 (2001) 42 .

[8] T. Whitaker, Opt. Laser Eur. 126 (March) (2004) 5.

[9] B.W. D'Andrate, R.J. Holmes, S.R. Forrest, Adv. Mater. 16 (2004) 624.

[10] T. Whitaker, Comp. Semicond. 10 (October) (2004) 20.

[11] S.R. Forrest, Org. Electron. 4 (2003) 45.

[12] B.W. D'Andrade, S.R. Forrest, Adv. Mater. 16 (2004) 1585.

[13] J. Kido, M. Kimura, K. Nagai, Science 267 (1995) 1332.

[14] R.S. Deshpande, V. Bulovic, S.R. Forrest, Appl. Phys. Lett. 75 (1999) 888.

[15] Z.Y. Xie, J.S. Huang, C.N. Li, S.Y. Liu, Y. Wang, Y.Q. Li, J.C. Shen, Appl. Phys. Lett. 74 (1999) 641.

[16] M. Strukelj, R.H. Jordan, A. Dodabalapur, J. Am. Chem. Soc. 118 (1996) 1213.

[17] R.H. Jordan, A. Dodabalapur, M. Strukelj, T.M. Miller, Appl. Phys. Lett. 68 (1996) 1192.

[18] F. Steuber, J. Staudigel, M. Stossel, J. Simmerer, A. Winnacker, H. Spreitzer, F. Weissörtel, J. Salbeck, Adv. Mater. 12 (2000) 130.

[19] S. Liu, J. Huang, Z. Xie, Y. Wang, B. Chen, Thin Solid Films 363 (2000) 294.
[20] C.W. Ko, Y.T. Tao, Appl. Phys. Lett. 79 (2001) 4234.

[21] K.O. Cheon, J. Shinar, Appl. Phys. Lett. 81 (2002) 1738.

[22] Y.S. Hunag, J.H. Jou, W.K. Weng, J.M. Liu, Appl. Phys. Lett. 80 (2002) 2782.

[23] B.W. D'Andrade, M.E. Thompson, S.R. Forrest, Adv. Mater. 14 (2002) 147.

[24] C.H. Chuen, Y.T. Tao, Appl. Phys. Lett. 81 (2002) 4499.

[25] G. Li, J. Shinar, Appl. Phys. Lett. 83 (2003) 5359.

[26] X.H. Zhang, M.W. Liu, O.Y. Wong, C.S. Lee, H.L. Kwong, S.T. Lee, S.K. Wu, Chem. Phys. Lett. 369 (2003) 478.

[27] S. Tokito, T. Iijima, T. Tsuzuki, F. Sato, Appl. Phys. Lett. 83 (2003) 2459.

[28] T.-H. Liu, Y.-S. Wu, M.-T. Lee, H.-H. Chen, C.-H. Liao, C.H. Chen, Appl. Phys. Lett. 85 (2004) 4304.

[29] G. Cheng, Y. Zhao, Y. Zhang, S. Liu, F. He, H. Zhang, Y. Ma, Appl. Phys. Lett. 84 (2004) 4457.

[30] D. Qin, Y. Tao, Appl. Phys. Lett. 86 (2005) 113507.

[31] Y. Xu, J. Peng, Y. Mo, Q. Hou, Y. Cao, Appl. Phys. Lett. 86 (2005) 163502.

[32] C.-T. Chen, Chem. Mater. 16 (2004) 4389.

[33] W.-C. Wu, H.-C. Yeh, L.-H. Chan, C.-T. Chen, Adv. Mater. 14 (2002) 1072.

[34] H.-C. Yeh, S.-J. Yeh, C.-T. Chen, Chem. Commun. (2003) 2632.

[35] H.-C. Yeh, L.-H. Chan, W.-C. Wu, C.-T. Chen, J. Mater. Chem. 14 (2004) 1293.

[36] C.-L. Chiang, M.-F. Wu, D.-C. Dai, Y.-S. Wen, J.-K. Wang, C.-T. Chen, Adv. Funct. Mater. 15 (2005) 231.

[37] J. Chen, B. Xu, X. Ouyang, B. Tang, Y. Cao, J. Phys. Chem. A 108 (2004) 7522.

[38] L.-H. Chan, H.-C. Yeh, C.-T. Chen, Adv. Mater. 13 (2001) 1637.

[39] L.-H. Chan, R.-H. Lee, C.-F. Hsieh, H.-C. Yeh, C.-T. Chen, J. Am. Chem. Soc. 124 (2002) 6469.

[40] T. Tsuji, S. Naka, H. Okada, H. Onnagawa, J. Appl. Phys. 81 (2002) 3329

[41] C.W. Tang, S.A. VanSlyke, C.H. Chen, J. Appl. Phys. 65 (1989) 3610

[42] G. Wyszecki, W.S. Stiles, Color Science, second ed., Wiley, New York, 1982, CIE 1931 is commonly used to describe the color of an light-emitting device. However, a uniform color space, such as CIE $1976 \mathrm{Luv}$ or Lab $\left(L^{*} u^{*} v^{*}\right)$ space, is needed for calculating CRI and color difference.

[43] C.-T. Chen, C.-L. Chiang, Y.-C. Lin, L.-H. Chan, C.-H. Huang, Z.-W. Tsai, C.-T. Chen, Org. Lett. 5 (2003) 1261. 